\title{
Study on Coagulation Effect of Composite Peel Coagulant in Treating Humic Acid Simulated Water
}

\author{
JIAN Hailong ${ }^{a}$, FU Ying ${ }^{*}$ \\ School of Civil Engineering and Architecture, University of Jinan, Jinan 250022, China \\ a1055801372@qq.com, bcea_fuy@ujn.edu.cn
}

\begin{abstract}
Keywords: Fruit peel; coagulant; humic acid simulated water; coagulation effect
Abstract. A composite fruit peel (HFP) coagulant was prepared from banana peel and orange peel, for humic acid simulated water, the effect of dosage and $\mathrm{pH}$ on the turbidity and color removal rates were investigated. The results showed that the HFP has $97.14 \%$ turbidity removal and $88.7 \%$ of color removal under the better dosage. HFP has good treatment effect on raw water under strong acid and alkaline conditions.
\end{abstract}

\section{Introduction}

Coagulation is a common water treatment process, which is economical and simple, and the most important factor for that is the effect of coagulant [1,2]. The requirements for water treatment process tend to be green and environmental-friendly, also the development and application of coagulants are moving closer to the direction of high efficiency, non-toxicity and easy degradation. Natural polymer coagulants have become more and more important due to their wide range of sources, low prices and excellent performance. China has a large fruit production and sales, and the negative factor is that a large amount of peels are abandoned considered as wastes, which pollutes the parts of environment [3]. Such as orange peel, grapefruit skin, banana peel, etc., these peels contain pectin, cellulose, lignin and other components, and in these valuable molecular structures exist carbonyl, carboxyl, hydroxyl and other active groups $[4,6]$. These active groups have a certain adsorption effect on small molecules of contaminants in water. A large number of scholars have conducted extensive studies on the preparation of adsorbents and coagulants for fruit peels. Zhang Jie applied the treated orange peel particles to the flocculation process of the drilling fluid. Through simulation tests, they found that orange peel particles have a better flocculation effect on the drilling fluid, and the best dosage of orange peel is $3 \%$, showing the best flocculation effect at $40^{\circ} \mathrm{C}$ [7]; Lu Ningning has used a coagulant prepared from banana peel,it has a good effect on the turbidity removal in humic acid simulated water sample and clay simulated water sample under certain dosage [8]. These studies have shown that the fruit peel has a great potentiality in terms of water purifiers preparation, not only can we treat sewage, but also we can consume pericarp waste to reduce the pressure of environment pollution.

In this article, coagulation experiments were performed using coagulants prepared from banana peel mixed with orange peel. The turbidity and color removal effects of coagulant simulated humic acid were investigated.

\section{Experimental materials and methods}

Instruments and Reagents. Instruments: Crusher (COSUA/Super Manipulator), ZR4-6 Coagulation Test Mixer (Zhongrun, Shenzhen, China), AUY120 Electroanalytical Balance (Shimadzu Instruments), 2100AN Desktop Turbidimeter (Hash, America), GXZ-9140MBE Significant blast drying oven (Boxun Industrial, Shanghai, China), SH05-3 thermostatic magnetic stirrer (Mei yingpu, Shanghai China), FE20 desktop PH meter (Mettler-Toledo, Switzerland).

Reagents: kaolin (Ke Miou, Tianjin, China), humic acid (Guangfu, Tianjin, China), sodium hydroxide (Damao, Tianjin, China), banana peel, and orange peel (Fruit shop, Jiannan, China).

Preparation of HFP. Orange peel and banana peel source: Fruit shop, the source of the fruit peel is relatively stable, after two days of natural air drying, and then oven drying at $70{ }^{\circ} \mathrm{C}$ for $24 \mathrm{~h}$. The 
powder was pulverized with a pulverizer and the peel powder was passed through a 100 mesh sieve for use.

A total of $1.25 \mathrm{~g}$ of orange peel and banana peel powder was weighed according to a certain proportion, and a certain concentration of sodium hydroxide aqueous solution was added, and the mixture was stirred at medium speed for 3 minutes. After stirring, it is placed in a $60^{\circ} \mathrm{C}$ water bath and leached for 10 minutes, then filtered. A certain amount of stabilizer is added to the filtrate to obtain the final medicament.

Coagulation experiments.

Preparation of humic acid simulated water sample. Preparation of humic acid simulated water sample: Take $450 \mathrm{~mL}$ of kaolin stock solution and $450 \mathrm{~mL}$ of humic acid stock solution respectively and add it to $20 \mathrm{~L}$ of tap water, stir for $15 \mathrm{~min}$, make humic acid simulation water sample

Raw water quality indicators:

Table 1 Test water quality

\begin{tabular}{cccccc}
\hline Sample Water & Turbidity[NTU] & Chroma [A] & COD [mg/L] & pH & Temperature $\left[{ }^{\circ} \mathrm{C}\right]$ \\
\hline $\begin{array}{c}\text { Humic acid } \\
\text { simulation water }\end{array}$ & $36.5-44.2$ & $0.334-0.385$ & 8.42 & $8.43-8.87$ & $20-27$ \\
\hline
\end{tabular}

Kaolin and humic acid stock solutions are prepared as follows:

Kaolin stock solution: $15 \mathrm{~g}$ of kaolin was added to $1 \mathrm{~L}$ of distilled water, stirred for $2 \mathrm{~h}$, allowed to stand for $3 \mathrm{~h}$, and finally $500-600 \mathrm{~mL}$ of the supernatant was used as a stock solution.

Humic acid stock solution: $1 \mathrm{~g}$ humic acid is added to a $0.01 \mathrm{~mol} / \mathrm{L}$ sodium hydroxide solution, and the solution is stirred magnetically for $2 \mathrm{~h}$ to obtain a standard stock solution with a humic acid concentration of $1 \mathrm{~g} / \mathrm{L}$ and stored at $4^{\circ} \mathrm{C}$.

Coagulation process. The speed of 200rpm was quickly stirred for $1 \mathrm{~min}$, then slowly stirred at 60rpm for $10 \mathrm{~min}$, then slowly stirred at $40 \mathrm{rpm}$ for $5 \mathrm{~min}$, and after stirring was completed, it was precipitated for $15 \mathrm{~min}$. Finally, take an appropriate amount of water sample from the outlet to measure, and determine the turbidity and color of the water sample.

Take 1L of humic acid simulated water sample for each of the six beakers on the six-joint blender, add the coagulant, and perform the experiment according to the above coagulation procedure.

Coagulant dosage test: Coagulant dosage was adjusted to 5, 15, 25, 35, 45, $55 \mathrm{mg} / \mathrm{L}$ for coagulation experiments to study the optimal dosage of coagulant.

Influence of raw water $\mathrm{pH}$ value: The $\mathrm{pH}$ of humic acid simulated water samples was adjusted to 3, $5,7,9,11,13$ and the dosage was selected to be the best dosage $25 \mathrm{mg} / \mathrm{L}$. The effect of $\mathrm{pH}$ value of raw water on the coagulation effect was studied.

\section{Results and Discussion}

Effect of coagulant dosage. Figure 1 shows that the turbidity removal rate and chromaticity removal rate both show a state of first increase and then stabilize with the increase dosage of coagulant, and at a lower dosage, the turbidity removal rate reached $90 \%$, and the color removal rate was close to $80 \%$. When the dosage of coagulant reaches $25 \mathrm{mg} / \mathrm{L}$, the removal of color and turbidity reaches a higher level. After that, when the dosage of coagulant increases, the turbidity and color removal rates do not increase significantly. The coagulation effect of the coagulant at a dosage of $25 \mathrm{mg} / \mathrm{L}$ reached a better level. 


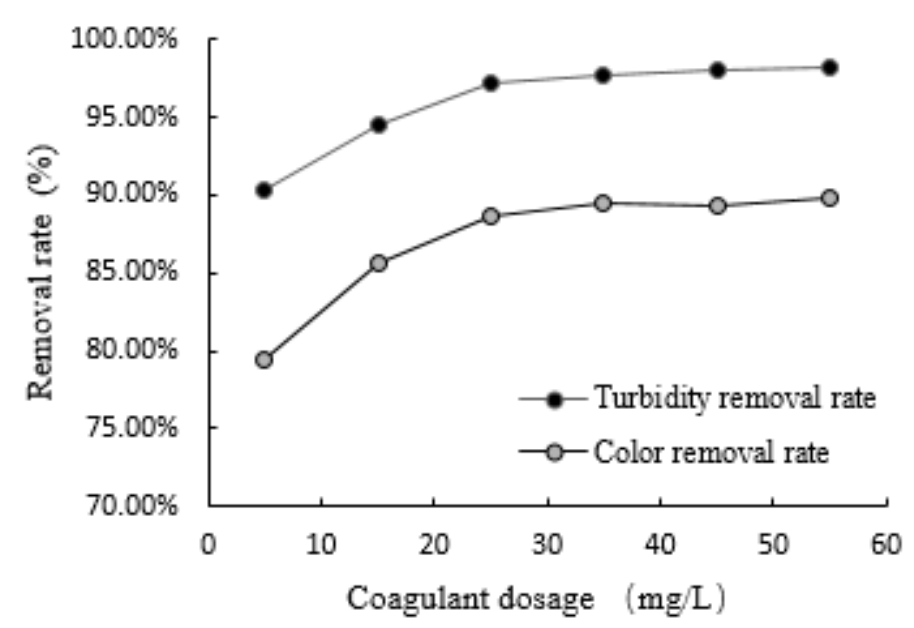

Fig. 1 Effect of Coagulant Dosage on Turbidity and Chromaticity

Effect of raw water pH. Figure 2 shows the effect of $\mathrm{pH}$ on turbidity and color removal:

The removal rate of turbidity is greatly reduced at 3-5, and increases sharply at 5-7. When PH is greater than 7, the removal rate of turbidity decreases slightly. The turbidity removal rate reached $97.79 \%$ at PH 9 and the removal rate of turbidity reached $96 \%$ at $\mathrm{PH}$ values of 3, 7, 11 and 13 .

Chromaticity removal rate drops sharply at 3-5, increases sharply at 5-7, and decreases slightly between 7-9, and the rate has gradually increased at 9-13, the overall fluctuations are more frequent; the chroma removal efficiency reaches an optimum of $95.56 \%$ at $\mathrm{pH} 13$ and the color removal efficiency exceeds $90 \%$ at $\mathrm{pH}$ values of 3,7 , and 11 respectively.

Combining the effects of turbidity and color removal from figure 2: When the acidity of the raw water is relatively large, or in an alkaline environment, the coagulant treatment performance has good performance, and the performance in the weak acid environment is not ideal. In general, the applicable $\mathrm{PH}$ range of coagulant is still relatively wide.

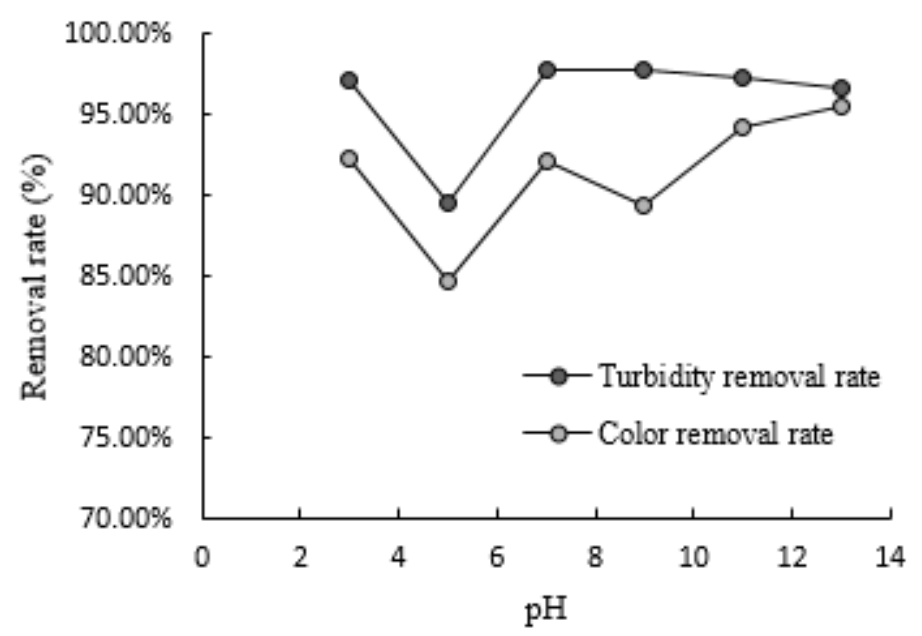

Fig. 2 Effect of Raw Water pH on Turbidity and Chromaticity

\section{Conclusion}

A composite peel coagulant was prepared by modifying the banana peel and the orange peel, and we obtained good experimental results. The turbidity and color removal of simulated humic acid wastewater have better effect at lower dosage, when the dosage of coagulant reached the best dosage, the removal rate of turbidity and color increased significantly, which were $97.14 \%$ and $88.7 \%$ respectively. The coagulant can be applied to strong acid, alkaline water quality, and has a good treatment effect. 


\section{Acknowledgements}

This work was financially supported by Shandong higher school science and technology project (L15LG04) and Joint Project between University and Company (W17121).

\section{References}

[1] X. H. Lu: Chemical Industry Environmental Protection vol. 15 (1995), p. 211.

[2] Z. Yang, B. Yuan, X. Huang. et al: Water Research vol. 46(2012), p. 107.

[3] B. B. Ma: Wuhan: Huazhong Normal University (2001), p. 12.

[4] S. Liang, X. Y. Guo and N. Feng, et al: Colloids and Surfaces B: Biointerfaces vol. 73(2009), p. 10.

[5] S. Liang, N. C. Feng and X. Y. Guo: Water Treatment Technology vol. 35(2009), p. 13.

[6] J. Sun, L. Li and X. R. You: Analytical Methods vol. 3(2011), p. 2212.

[7] J. Zhang, X. F. Chang and G. Chen: Ecotechnology vol. 6(2016), p. 1.

[8] N. N. Lu: Jinan: University of Jinan (2017), p. 28. 